\title{
Tangence
}

\section{De la référence au reflet : la semiosis du vraisemblable dans le théâtre de recherche}

\section{Dominique Lafon}

Numéro 46, décembre 1994

Un théâtre de passage

URI : https://id.erudit.org/iderudit/025837ar

DOI : https://doi.org/10.7202/025837ar

Aller au sommaire du numéro

Éditeur(s)

Tangence

ISSN

0226-9554 (imprimé)

1710-0305 (numérique)

Découvrir la revue

Citer cet article

Lafon, D. (1994). De la référence au reflet : la semiosis du vraisemblable dans le théâtre de recherche. Tangence, (46), 20-28. https://doi.org/10.7202/025837ar d'utilisation que vous pouvez consulter en ligne.

https://apropos.erudit.org/fr/usagers/politique-dutilisation/ 


\section{De la référence au reflet: la semiosis du vraisemblable dans le théâtre de recherche Dominique Lafon}

De tous les arts de la représentation, c'est sans doute le théâtre qui pose avec le plus d'acuité le problème de la vraisemblance. Si la vraisemblance du récit théâtral se satisfait pour une part des modalités de la logique narrative, modalités que les classiques formulaient en termes d'unité d'action ou de péril, les conditions de sa mise en scène sont au cœur de toutes les ambiguités dont se nourrit le concept aristotélicien de mimesis, concept que la sémiotique analyse en termes d'illusion référentielle. L'illusion référentielle est, au théâtre et sans doute seulement au thêâtre, outre un objet d'analyse, une pratique concrète dont l'artisan héroïque est, plus que le metteur en scène, le comédien. La personne du comédien saisie par le spectateur dans l'interprétation du personnage est la figure emblématique de la paradoxale conciliation que tente chaque représentation, conciliation du vrai et du faux, de la présence et de l'absence, du signe motivé et du signe arbitraire. L'histoire thêâtrale ne consacre-t-elle pas comme divinités tutélaires dont elle ne cesse d'interroger le mystère, ces auteurs-acteurs qu'on appelle Molière ou Shakespeare justement parce que leur oeuvre concilierait la pratique du geste et la maîtrise du texte? Consécration de l'histoire, mais aussi fascination des metteurs en scène contemporains dont la carrière se mesure aux ré-interprétations qu'ils n'ont de cesse d'en soumettre. C'est Planchon, Vitez en France, c'est Lepage au Québec dont Le Festival des Amériques présentait naguère pas moins de trois mises en scène de Shakespeare. Ce dernier événement dont l'actualité et l'excès autorise qu'on le juge exemplaire, permet de formuler les composantes de la modernité théâtrale, on dirait ailleurs de la post-modernité, en terme de semiosis du vraisemblable. Car il ne s'agit plus seulement d'actualiser, comme dans certaines tentatives où la modernité se résumait à un anachronisme du costume, mais bien d'appliquer au texte, en l'occurrence réécrit plus que traduit par Michel Garneau, un ensemble de signes plastiques, de signifiants essentiellement visuels. Faut-il 
voir là une pratique de la modernité, celle là même que Kowzan ${ }^{1}$ définit comme l'association de "signifiants nouveaux à des signifiés habituels " ou encore comme le bouleversement "des rapports établis entre les signes et leurs référents", autrement dit une nouvelle semiosis du vraisemblable? Cette pratique parvient-elle à éviter l'impasse de ce "jeu complexe et quelquefois insolite, entraînant les signifiants, les signifiés et les référents de signes ou d'entités signifiantes dans un tourbillon vertigineux", impasse que ce même auteur décrit comme "un désert sémantique"? Ces questions qui interrogent, on l'aura compris, plus une mode que la théorie sémiotique du vraisemblable, trouvent cependant dans les modalités de l'analyse sémiotique qui prend en compte le signe, l'objet (extérieur au signe) et l'interprète (l'interprétant), l'outil privilégié de leur articulation.

Interroger la mode, c'est bien sûr interroger le succès, l'événement. Aussi, ai-je choisi, comme objets d'analyse, trois praticiens dont la réputation internationale n'est plus à faire ou est en train de se faire, à savoir Ariane Mnouchkine, Robert Lepage et Gilles Maheu. Outre le fait que leur production présentait l'avantage d'être ou d'avoir été accessible à mon propre public-lecteur et suffisamment récente pour être encore présente à son esprit, leur parcours personnel semble, toutes considérations chronologique ou géographique mises à part, les unir. Ce sont trois metteurs en scène dont l'activité s'exerce à l'intérieur, ou plutôt à la tête, d'un collectif. Le terme de collectif convient mieux que celui de troupe tant il est patent qu'ils affirment chacun différemment, mais non sans une certaine similarité, la nature collégiale, voire familiale dans le cas de Mnouchkine - c'est une femme, d'un groupe dont la pratique ne saurait se limiter au seul exercice de la répétition d'un spectacle. Le Théâtre du Soleil, le groupe Repère, Carbone 14 qui est issu des Enfants du Paradis sont, plus que des titres, les signes d'une identité collective qui se manifeste dans la permanence des membres qu'elle regroupe. C'est si vrai qu'un acteur du Théâtre du Soleil a pu, ou a dû en expliquer son départ en présentant un one man show qui ne racontait rien d'autre que la geste du groupe. Mais au delà de l'histoire particulière de ses groupes, qu'elle soit mythique ou

1 Tadeusz Kowzan, "Avant-garde, modernité, créativité : jeu insolite entre signifiants, signifiés et référents", dans Spectacle et signification, Candiac, Éditions Balzac, coll. "L'Univers des discours", 1992, p. 181-206. 
non, il y a, dans leur origine, une volonté commune que l'on pourrait caractériser comme une revendication fondamentale: promouvoir le jeu de l'acteur; et pour cela concevoir des spectacles en fonction du jeu de l'acteur, du geste de l'acteur. Ces groupes ont ainsi recours à l'improvisation, Lepage est d'ailleurs une ancienne étoile de la Ligue nationale d'improvisation, au mime, la formation de Gilles Maheu s'est faite chez Decroux. Les spectacles du Théâtre du Soleil sont tout entier conçus autour d'un code gestuel caractéristique d'une pratique spectaculaire: bateleurs, commedia dell'arte, clowns... Ils partagent, enfin, la même conception de la nécessaire maturation du spectacle, ce qui les contraint à de longs processus de conception, ou leur fait assumer, dans le cas de Lepage, la modalité du work in progress, qui dégage les représentations de tout impératif d'achèvement ou de reproductibilité.

Ces groupes constituent donc un objet d'analyse relativement cohérent, symptomatique en tout cas d'une nouvelle conception de la représentation qui repose à l'évidence sur une prédominance du geste de l'acteur, prédominance qui a pour corollaire un traitement iconoclaste du texte et/ou une prise en charge de l'écriture textuelle. Le Théâtre du Soleil a longtemps conçu ses propres textes, puis a tenté une collaboration avec H. Cixous et est entré résolument dans l'appropriation des textes classiques. A. Mnouchkine a ainsi signé, non sans quelque audace, une nouvelle traduction des tragiques grecs à partir, il est vrai, d'un mot à mot fourni par un helléniste, et n'hésite pas à recomposer en partie le texte d'Eschyle, en particulier le rôle des chours. G. Maheu réduit à presque rien la part du discours dans Le rail ou Le dortoir, lui substituant dans Le café des aveugles, le chant, ou en exhibe le caractère marginal, en installant dans Peau, chair et os la figure conventionnelle de l'écrivain à l'une des extrémités de l'avant-scène. Ce spectacle, tout entier conçu autour d'un poème d'Heiner Müller, est l'aboutissement d'une recherche que G. Maheu a menée dans plusieurs spectacles antérieurs, Hamlet Macbine, Rivage à l'abandon, recherche qu'il définit comme un Werkscbau, équivalent allemand du work in progress, imposé selon lui par la matière même de l'écriture de Müller :

Je reviens sans cesse à Müller [...]. C'est à la fois exaltant et angoissant de travailler sur ces textes semblables à des parti- 
tions de musique concrète; je défie tous ceux qui se targuent de les connaitre par cœur de m'expliquer ce qu'ils veulent dire $!^{2}$

Cette citation résout pour une part, me semble-t-il, toute problématique du vraisemblable qui prétendrait prendre en compte la dimension textuelle. Les modalités de cette nouvelle écriture scénique, que l'on désigne de plus en plus couramment sous le terme de scénographie, sont d'abord et avant tout scéniques, s'inscrivent dans une esthétique de la Bildlichkeit, et ne sauraient être évaluées dans les rapports à un texte absent, ou recomposé, voire soumis au projet spectaculaire. Quel est donc le référent de cet ensemble de signifiants, qui repose moins sur une recherche du décor que sur la variation infinie des gestes de l'acteur, de ce kaléidoscope de la gestuelle qui refuse le support, la caution, le signifié textuel?

L'analyse des Atrides, dans la mesure où la production conserve des liens explicites avec le texte, permet de poser quelques balises préalables à celle de la semiosis scénographique. On se rappelle que la représentation de la tétralogie s'inscrit dans un seul et même espace scénique, sorte d'arène monochrome dont la conception évoque plus la corrida que le théâtre d'Epidaure et exhibe assez que la vraisemblance spectaculaire ne saurait $s$ 'appuyer sur la référence historique, référent qu'interdit, concédons le, l'impasse de toutes les reconstitutions des spectacles antiques, fussent-elles tentées dans les ruines mêmes de leurs lieux scéniques originels. C'est cette arène qu'investira le chour, dont les acteurs qui le composent seront les seuls à en utiliser toutes les possibilités spatiales, le jeu des protagonistes étant limité à l'espace central. Le chour dessine ainsi l'aire de jeu par la chorégraphie de ses déplacements, chorégraphie d'autant plus perceptible qu'elle est exaltée par la magnificence des costumes comme par l'intensité du maquillage des choreutes. En comparaison, le costume et le maquillage des protagonistes parait quelque peu neutre, tant dans son chromatisme que dans l'imprécision de leurs référents esthétiques. Car le chour, l'image comme les déplacements du chour, est une citation spectaculaire. En effet, un référent exhibé est-il autre chose qu'une citation, lui qui n'exige aucun décodage, sinon une reconnaissance, celle, en

2 Diane Parlovic, "Gilles Maheu, l'espace vital", Cabiers de théâtre Jeu, n 63 , 1992 , p. 20. 
l'occurrence, du kathakali. Cette reconnaissance d'une pratique gestuelle symbolique totalement étrangère à l'interprète-public occidental qui n'en possède ni le code, ni l'expérience ne saurait être analysée comme une des modalités de la vraisemblance. Au contraire, elle oblige le spectateur à une double dénégation: dénégation de la lecture historique du référent "Grèce antique", dénégation de la lisibilité du code "oriental". La citation visuelle et gestuelle ne saurait, dans ces conditions, engendrer de semiosis spectaculaire, mais tout au plus, une sorte de folklorisation de la tragédie grecque. Cette folklorisation est redoublée par l'omniprésence d'un accompagnement sonore qui, à la grande fierté de son compositeur, utilise les instruments traditionnels d'un grand nombre de cultures à l'exposition desquels le concept scénique consacre le tiers d'un espace scénique qu'il domine. Ce bric-àbrac sonore ne s'inscrit donc dans aucun code référentiel, pas plus qu'il ne constitue une trame sonore qui réaliserait une scansion du jeu gestuel. Ce bruitage, que sa constance rend paradoxalement monocorde, accompagne certes les gestes, mais couvre aussi les paroles, toutes les paroles uniformément. Il faut ajouter à ce bruitage, cette autre manifestation sonore que constitue l'accent des comédiens qui confère à la ligne mélodique de leur intonation, un caractère plus musical que discursif. Toutes ces modalités circonscrivent la majeure partie de la communication scène-salle dans un seul espace, celui du spectaculaire chorégraphique et musical. Dans le public, seuls quelques spectateurs, interprètes privilégiés, pourront établir les liens possibles qui unissent la ritualisation tragique et le symbolisme du kathakali; juger de la pertinence, qui n'est que le terme critique de la vraisemblance, qu'il y a à uniformiser les apparitions du chœur d'une pièce à l'autre, sans tenir compte des variations fictives spécifiées par le texte, voire inscrites dans le titre des ouvres, Les Choéphores, Les Euménides. Et là encore, il leur faudra négliger, sous peine d'incohérence, le bric-à-brac référentiel qui permet de voir dans Les Erynies une référence à La planète des singes, dans le costume de Cassandre une référence au Nô, dans l'installation, aux dénouements, des mannequins de cire sanguinolents, une référence au Grand guignol... Non, ce qui nourrit la vraisemblance de la tétralogie du Théâtre du Soleil, ce n'est ni le naturel, ni la cohérence des signifiants, telle qu'elle se manifestait dans les Shakespeare. Dans ces mises en spectacle, le recours au code du théâtre Nô s'appuyait en effet sur une métaphorisation des luttes dynastiques qui nourrissent les deux récits selon une relation 
complexe mais particulièrement féconde: le signifiant du Nô, signifiant conservé par une tradition ancestrale, spécifique des théâtres orientaux, réactivant le signifié du récit shakespearien dont la culture occidentale a perdu à jamais les modalitês signifiantes. Au delà d'une sémiotique du vraisemblable, les Shakespeare consacraient le projet d'une poétique de la théâtralité qui transcendait la contingence historique par l'exaltation du syncrétisme thêâtral, réalisant ainsi pour une part le projet artaudien d'un "théâtre pur " régi par "une intellectualité admirable que l'on sent crépiter partout dans la trame serrée et subtile des gestes [...] dans l'entrelacs lui aussi sonore des mouvements ". Cette citation extraite du chapitre sur le théâtre balinais qui, dans Le thêâtre et son double, exalte la technique du danseur plus que la signification d'un spectacle qu'Artaud décrit comme "les affres d'une âme en proie aux phantasmes de l'au-delà ", comme "une métaphysique du désordre. Elle permet aussi de formuler l'hypothèse suivante: ce qu'exaltent Les Atrides, c'est la geste du Théâtre du Soleil, celle-là même qui a conduit la troupe sur les traces des bateleurs, des clowns, qui l'a autorisée à interpréter l'histoire au travers du prisme du code théâtral - et aussi sans doute, à ses débuts du moins, à la lumière de la doxa de Mai 68 - celle qui la conduit aujourd'hui à s'approprier les textes fondateurs du théâtre occidental. Il ne faut pas compter pour rien dans cette perspective, la dramatisation de toute La Cartoucherie qui multipliait la rituelle et désormais familière exhibition des coulisses dans les artefacts d'une pseudo fouille archéologique où le spectateur était implicitement invité à voir les signes tangibles, les icones de la légitimité de l'entreprise spectaculaire. Légitimité, version morale et idéologique de la vraisemblance... Si, sous La Cartoucherie, on avait pu découvrir les vestiges authentiques du chœur grec pétrifié, si on pouvait presque, dans les coulisses de La Cartoucherie, toucher les corps des comédiens, saisis dans leur intimité comme dans leur mutation vers, comme dit Artaud, leur propre "effigie", sur le plateau de La Cartoucherie, monde autonome qui commande, par le jeu d'une verrière et de projecteurs placés à l'extérieur du bâtiment, sa propre lumière... référentielle, peut-il y avoir, sur ce plateau, autre chose que la consécration de ce montage, de ces stations, à savoir le vrai théâtre, le garant des codes du spectaculaire?

La jeune scénographie québécoise ne prétend pas constituer un tel musée vivant où l'acteur devient l'artefact de la gestuelle. 
Ces premières réalisations n'en furent pas moins ambitieuses: $L e$ rail, Le dortoir, La trilogie des dragons, Les plaques tectoniques ont pour but explicite, avoué de saisir l'histoire, voire la dérive des cultures. Véritables fresques, elles s'inscrivent dans l'esthétique des spectacles-fleuves, des cycles. Mais, à la différence des premières créations de Mnouchkine, elles ne s'appuient pas sur un code théâtral spécifique, mais sur une pratique gestuelle originale dont la caractéristique prédominante est la plasticité. Si on a déjà caractérisé le signe théâtral par sa mobilité ${ }^{3}$, c'était d'abord pour rendre compte de la gamme des possibilités référentielles d'un signifiant scénique. Substituer plasticité à mobilité, c'est rendre compte non seulement de la volonté chorégraphique, voire acrobatique qui se manifeste dans les productions de G. Maheu, mais aussi, et surtout des modalités de la réception qui, dans l'écriture scénographique, permettent d'assimiler le processus encodage-décodage à un changement à vue dans lequel l'acteur joue le rôle d'un manipulateur.

Deux exemples, mais on pourrait les multiplier, permettront d'illustrer ce point. Dans le dernier tableau de La trilogie des dragons de Robert Lepage, une installation lumineuse faite d'une chaîne de minuscules ampoules, dénote, en un premier temps l'œuvre que le héros expose dans sa galerie d'art de Vancouver; en un second temps, alors qu'un acteur mime le décollage d'un avion du haut d'une chaise, l'éclairage change permettant à la chaîne lumineuse de reproduire les lumières de la ville, telles qu'on peut les percevoir, de nuit, du haut d'un avion. Ce qui est donné à voir, plus qu'à décrypter, c'est la métamorphose, la transformation du point de vue que seul, le corps de l'acteur spatialise. Ce que les spectateurs applaudissent, car ils applaudissent toujours à ce moment précis, c'est bien un tour de passe-passe, un numéro de prestidigitation qui ne s'exerce qu'au niveau du signifiant et de la dénotation.

Dans Les aiguilles et l'opium, Robert Lepage exhibe sa virtuosité de manipulateur dans les variations qu'imposent à l'écran, élément central du dispositif scénique, les différentes positions de son corps dans l'espace. Cette exhibition culmine dans l'imitation saisissante de Jean Cocteau, lui aussi touche-à-tout de génie, que tout le spectacle, construit pour une part autour de citations de

3 J. Honzl, "La Mobilité du signe théâtral", Travail théâtral, 4, 1971, p. 19 et sq. 
l'œuvre, qu'elle soit littéraire, graphique ou cinématographique, érige en figure emblématique. Ce qui serait ailleurs une référence à Cocteau, ou une citation, est, par le biais de l'imitation, une appropriation de l'image. Quand Lepage cite Cocteau, c'est livre en main, s'étant fait de plus la tête de Cocteau, ayant pris sa célèbre voix nasillarde. Le référent est ainsi cautionné par la composition physique, par le jeu de l'acteur dont la virtuosité se donne libre cours sur le mode, là encore du changement à vue, de la manipulation; à preuve, cette séquence où la citation du texte s'accompagne d'une citation-reproduction de la photo parue dans le magazine Life et qui, en un judicieux montage, dote Cocteau de quatre bras. Le spectateur, qui perçoit simultanément la projection de la photo sur l'écran et l'animation qui en est faite par Lepage, ne peut évaluer, l'image ne renvoyant qu'à l'image, que le signifiant scénique, la virtuosité dénotative de la gestuelle. L'exercice spectaculaire ne saurait là encore relever d'une sémantisation; il s'agit bien plutôt d'une exhibition de la plasticité du signifiant, en l'occurrence le corps de l'acteur, et, si l'on en juge par l'ensemble du one man show que sont Les aiguilles et l'opium, d'une exhibition de la personne de l'acteur. Car le fil conducteur des créations de la nouvelle scénographie québécoise, et particulièrement des plus récentes, repose sur la confidence autobiographique. Qu'il s'agisse du récit explicite d'un chagrin d'amour dans Les aiguilles et l'opium, ou, dans Le café des aveugles, de la lecture, en scène et en personne, des lettres que Maheu adressa à ses parents, le propos est le même: cautionner le vraisemblable par l'authentique, la représentation par l'exhibition, le signe arbitraire par la motivation personnelle. N'est-ce pas, en fin de compte, l'aboutissement logique d'une pratique esthétique qui travaille tout entière à réduire dans la perception du spectateur l'écart entre le signifiant scénique et son référent simultanément présenté ou, après un changement à vue, présenté à son tour comme signifiant scénique. De même, l'effigie de l'acteur se substitue-t-elle à la figure du personnage, en un processus qui tend à réduire non seulement l'interprétation à une virtuosité du geste, mais aussi et surtout, l'interprétation du spectateur à la passivité d'un voyeur... d'images dont on serait tenter de dire qu'elles ne sont que des instantanés ou, poussant plus loin la critique, des clichés.

Cette paradoxale semiosis de l'acteur qui s'élabore du signifiant au signifiant, où le mimétique fait place au mimique, le vrai- 
semblable au véridique, connaît, c'est incontestable, un très grand succès. De ce succès l'avenir jugera, dira la part qu'y prit la mode; son ampleur nous impose cependant de l'observer comme, selon la formulation de P. Pavis, un moment caractérisé par une évaluation spécifique du vrai et du semblable et donc comme une conception du vraisemblable: "Au lieu de juger une fois pour toutes le fictionnel et le vrai [...], il vaut mieux observer quels codes idéologiques sont en vigueur à un moment donné, [...] quel système du vraisemblable régit la description de la réalité sociale. "4 À la lumière de ce programme, les phénomènes spectaculaires contemporains s'inscrivent dans un courant dont on peut d'ores et déjà, et malgré l'absence de recul, relever quelques traits. Sa première caractéristique, celle de l'exaltation du travail de l'acteur malgré ou sans texte, marque l'aboutissement, et peut être le terme, de toute la réflexion théorique de notre époque qui, prétendant fédérer texte et représentation, a, en fait, sacrifié celui-ci à celle-là. Si du "je pense donc je suis", on était passé au "je lis donc je suis", on en est aujourd'hui au "je joue donc je suis ", sans qu'il soit possible, mais peut-être n'est-ce que partie remise, de poursuivre le syllogisme jusqu'au "je joue donc je pense"... Les implications sociologiques d'une telle évolution, qu'évoquait implicitement la mise en parallèle du Théâtre du Soleil et des scénographes québécois, me paraissent assez manifestes pour qu'il ne soit pas nécessaire de les formuler en des termes qui risqueraient bien de n'être, eux aussi, que des clichés. La seconde caractéristique de ces manifestations spectaculaires, à savoir la primauté d'une succession accélérée d'images, traduit la nécessité dans laquelle les praticiens du théâtre, ou du moins d'un certain thêâtre, se croient placés et qui les contraindrait à rivaliser avec les techniques de l'image dont notre société fait, il est vrai, une prodigieuse consommation de tous les instants. Il y a de moins en moins d'interprétants potentiels dans le public d'une salle de théâtre, et de plus en plus de spectateurs captifs de l'image. Alors, à tout prendre, aller voir Lepage dans Lepage, c'est peut-être une étape nécessaire pour inciter le public à aller voir Shakespeare par Lepage. Comme si, pour séduire, il suffisait d'en avoir fini avec un certain vraisemblable...

4 Patrice Pavis, Voix et images de la scène, pour une sémiologie de la réception, Presses Universitaires de Lille, 1985, p. 280. 